\title{
Optimization Studies of a Water Cherenkov Detector for Gamma-ray Astronomy
}

\author{
Samridha Kunwar*, Harm Schoorlemmer, Jim Hinton \\ Max Planck Institut für Kernphysik, Saupfercheckweg 1, D-69117 Heidelberg, Germany \\ E-mail: samridha.kunwar@mpi-hd.mpg.de
}

\begin{abstract}
Ground-based, wide field of view instrumentation in gamma-ray astronomy is currently limited to the northern hemisphere and hence, lacks sensitivity to our Galactic Center and the rest of the southern sky. A Southern Gamma Observatory comprising an array of ground-level detector units with a high fill factor $(>70 \%)$ at high altitude $(>4 \mathrm{~km})$ could be used to observe the southern sky. Water Cherenkov Detectors (WCDs) have shown their potential as detector units for such an array. Detailed simulations are needed to understand the response of WCDs to the flux of secondary particles impinging on them. In this contribution, we study the complete simulation chain from the air shower simulation with CORSIKA to the detector level simulation with GEANT4, in an attempt to optimize WCDs.
\end{abstract}

36th International Cosmic Ray Conference -ICRC2019-

July 24th - August 1st, 2019

Madison, WI, U.S.A.

\footnotetext{
${ }^{*}$ Speaker.
} 


\section{Introduction}

As a result of the declining flux emitted by cosmic sources, at Very High Energies (VHE) of $\sim 100 \mathrm{GeV}-\sim 100 \mathrm{TeV}, \gamma$-rays can be best observed by ground-based detectors through their induced electromagnetic cascade in the atmosphere. There are two approaches to observe these particle cascades: Recording Cherenkov light emitted by these cascade particles with Imaging Atmospheric Cerenkov Telescopes (IACTs), or observing the cascade particles directly with particle detectors. IACTs need dark and cloud-less conditions resulting in a low duty cycle of $\sim 1000$ hours/year while telescope optics and camera size result in small Field-Of-View (FOV) of $<10^{\circ}$. Additionally, increasing the high energy limit requires an increased collection area, which can be prohibitively expensive. Particle detectors, on the other hand, are relatively inexpensive, intrinsically wide FOV and have $\sim 100 \%$ duty cycle. High altitude $(>4 \mathrm{~km})$ and a high fill factor $(>70$ $\%$ ) allow such detectors to be complimentary in the same energy range as IACTs.

Currently, there are two ground particle detectors in operation. Both HAWC [1] on the flanks of the Sierra Negra volcano in Mexico and LHAASO [2] in the eastern Tibetan plateau comprise an array of Water Cerenkov Detector (WCD) units with Photo-Multiplier Tubes (PMTs) that are triggered by Cherenkov photons produced by the passage of secondary particles impinging on them. These instruments are providing compelling results in $\mathrm{TeV} \gamma$-ray astronomy, however their location in the northern hemisphere limits sensitivity to our Galactic Center and the rest of the southern sky. An array in the southern hemisphere aims to provide unprecedented observations of interesting phenomena in the TeV $\gamma$-ray sky [3]. An inner array with a high fill factor of $>70 \%$ will enhance sensitivity for sub-TeV $\gamma$ rays while an outer array with a fill factor of $8 \%$ in Fig 1 aims to improve sensitivity at higher energies. Air shower simulations with COR-

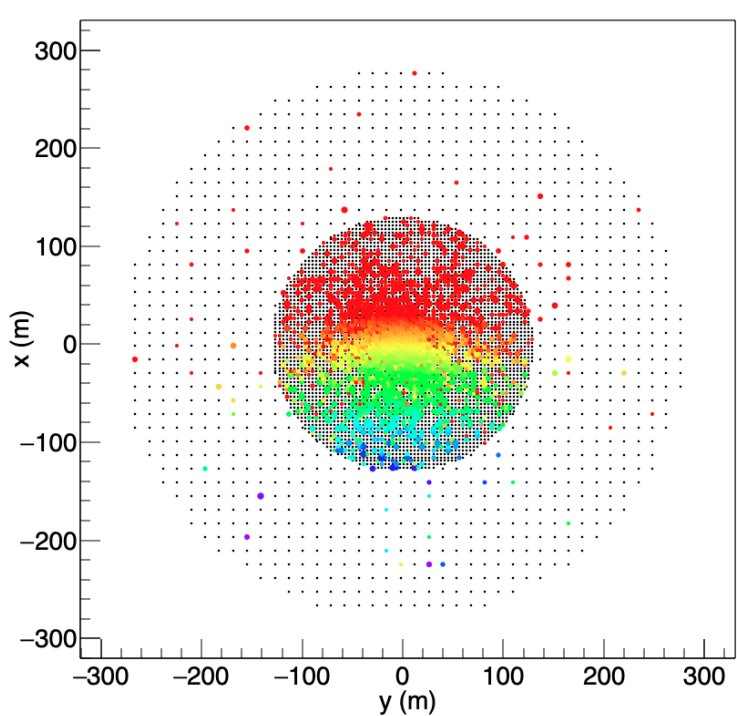

Figure 1: Top view of a $\gamma$-ray observatory comprising an inner and outer array of $>70 \%$ and $8 \%$ fill factor respectively. This illustration of a $2000 \mathrm{GeV}$ $\gamma$-ray air shower at a $0^{\circ}$ zenith angle incident on the array shows photo-electrons (p.e) collected at individual WCD units, where the color indicates an arrival time gradient. SIKA (COsmic Ray SImulations for KAscade) [4] and detector level simulation with GEANT4 (for GEometry ANd Tracking) [5] have been performed to improve on WCD designs similar to HAWC.

\section{Design Overview}

Here we describe a possible detector concept (Fig. 2) that comprise the following building blocks: 


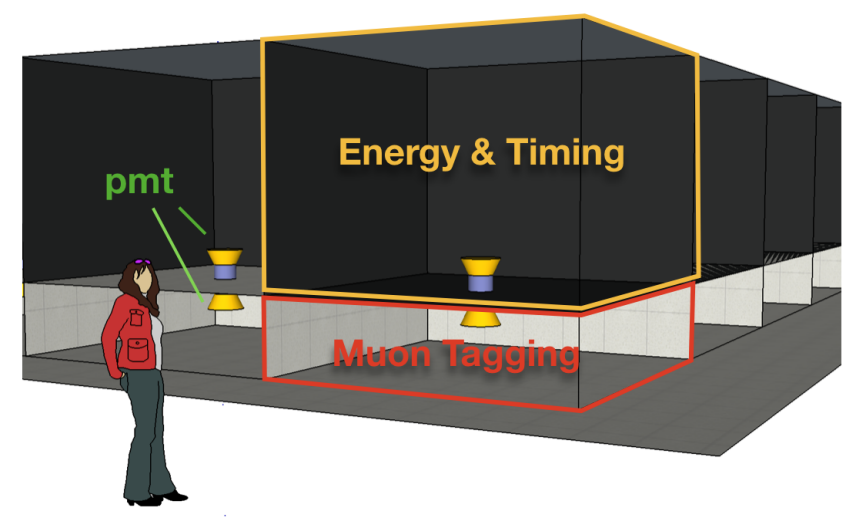

Figure 2: The illustration shows a conceptual design of a detector unit. Each WCD unit comprises an upper and lower chamber with a light sensor in each chamber.

- Upper Chamber. A light-tight chamber with a reflective lining comprising a single light sensor facing upwards. This chamber provides the energy and timing of the impinging particle where the upward facing light sensor ensures that the direct Cherenkov photons are detected first.

- Lower Chamber. A light-tight chamber with a highly reflective lining and comprising a single light sensor facing downwards. This chamber will enable muon tagging as only a small fraction of the higher energy photons and electrons at ground level can punch through into the lower chamber, while most muons will also deposit photons in this chamber.

\section{Simulation}

At ground level, for $\gamma$-ray induced extensive air showers (EAS), the peak of the number density distribution in terms of number per log energy interval ( $\mathrm{dN} / \mathrm{dlog} \mathrm{E}$ ) is around $\sim 6 \mathrm{MeV}$ for photons, $\sim 20 \mathrm{MeV}$ for electrons and 2-3 GeV for muons [6]. GEANT4 within the HAWC-SIM framework used by the HAWC collaboration [1] enables running simulations of such particles striking the WCD units. In this study, we first optimize individual units by varying the dimensions and the reflectivity of the material lining the walls of the two chambers for the different particle species. In Fig. 3, on the left, we illustrate a single muon entering a WCD unit with highly reflective upper and lower chamber wall linings and, on the right, a $\gamma$-ray enters an identical unit. The $\mu$ and $\gamma$-ray energies were chosen to illustrate a similar number of photons deposited in the upper chamber of the WCD unit. Low energy $\gamma$-rays do not punch through into the lower chamber.

In simulating air showers, the Monte Carlo uses the CORSIKA 7.4005 simulation package [4]. For the standard simulated event set, we selected QGSJet-II [7] for energies above $80 \mathrm{GeV}$ and FLUKA [8, 9] hadronic models for below $80 \mathrm{GeV}$ energies, respectively. For electromagnetic processes, the EGS4 electromagnetic model [11] was used. 

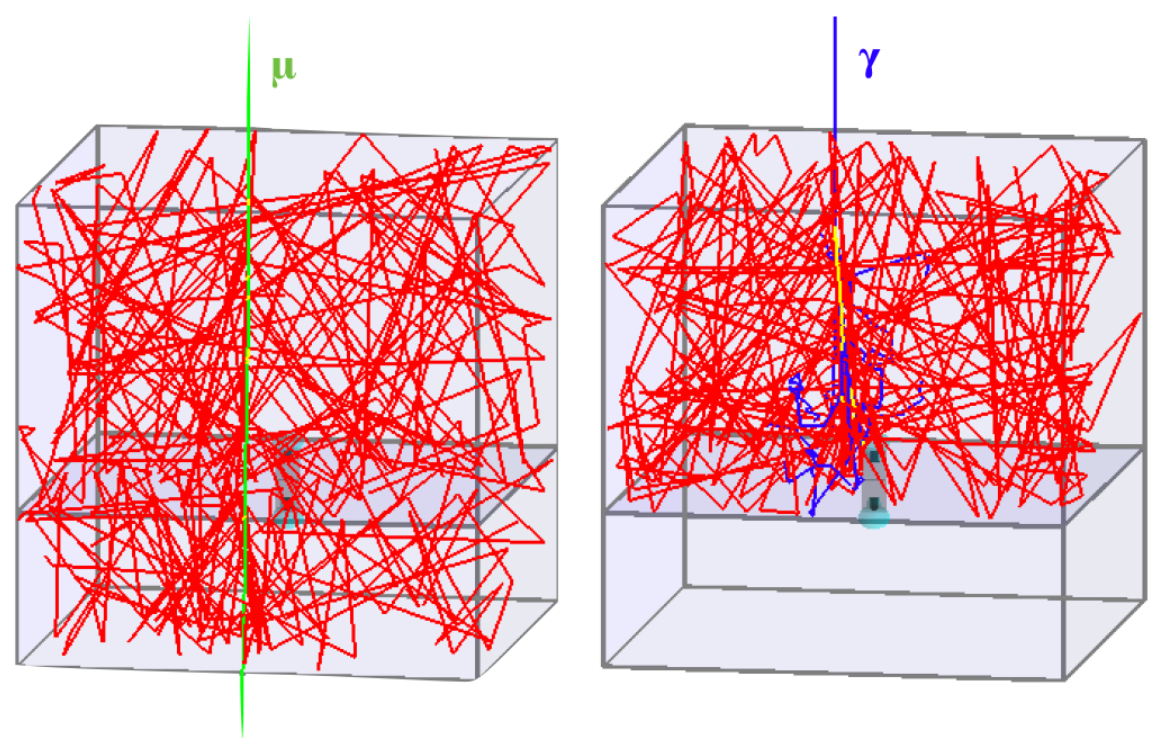

Figure 3: The illustration shows a muon (green track) and a $\gamma$-ray (blue track) depositing similar amount of Cherenkov photons (red tracks) after entering WCD units comprising highly reflective wall linings.

\section{Results}

\subsection{Reflectivity}

$10 \mathrm{MeV} \gamma, 3 \times 3 \times 2 \mathrm{~m}$ Upper Chamber, 8 inch PMT
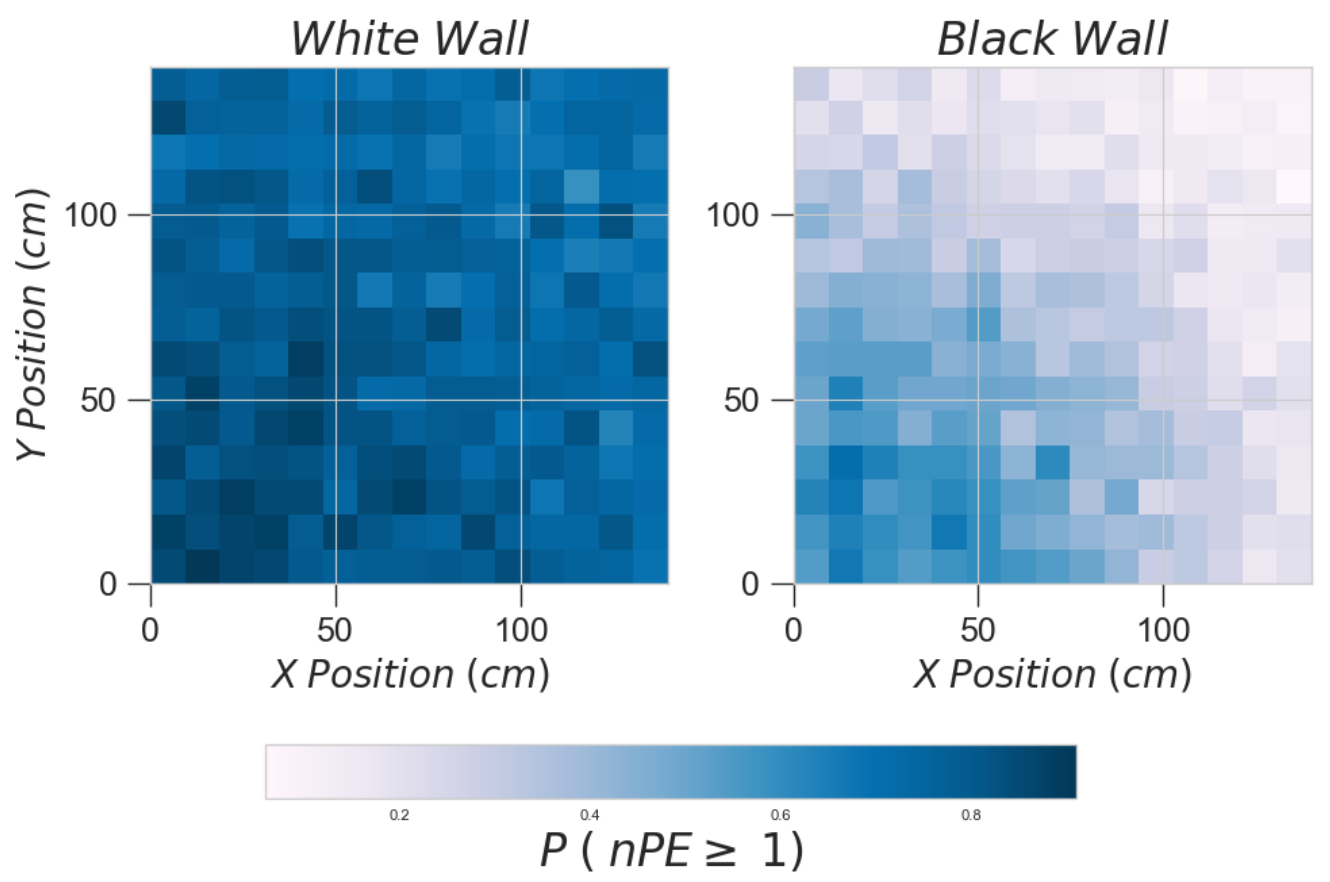

Figure 4: Scan of the upper chamber of a WCD unit with vertical $10 \mathrm{MeV} \gamma$-rays with white walls on the left and black walls on the right. The PMT is located at $(0,0)$. 
In order to investigate the effect of wall reflectivity on the probability of the detection of photons at a light sensor, vertical $\gamma$-rays at $10 \mathrm{MeV}$ energies were used to scan a quadrant of the surface of the upper chamber of $3 \times 3 \times 2$ m dimensions in a WCD unit.

Walls with a rough top layer of either high reflectivity (white) like Tyvek as used by the Pierre Auger Observatory [10] or low reflectivity (black) like Polypropylene as used by HAWC have been selected (see Fig. 4). An 8 inch PMT was the light sensor in this study. Due to the angle of the Cherenkov cone in water, the black walls result in a reduced sensitive area.

\subsection{Timing}

Time degradation arises from late photons arriving at the light-sensor with a whitewalled compared to a black-walled upper chamber. $\gamma$-rays with energy ranging from $1 \mathrm{MeV}$ to $1 \mathrm{GeV}$ were used to scan the surface of a WCD unit with an upper chamber of $3 \times 3 \times 2$ m dimensions. The first p.e's arriving at the light-sensor will be used for timing; however, further investigation is neces-

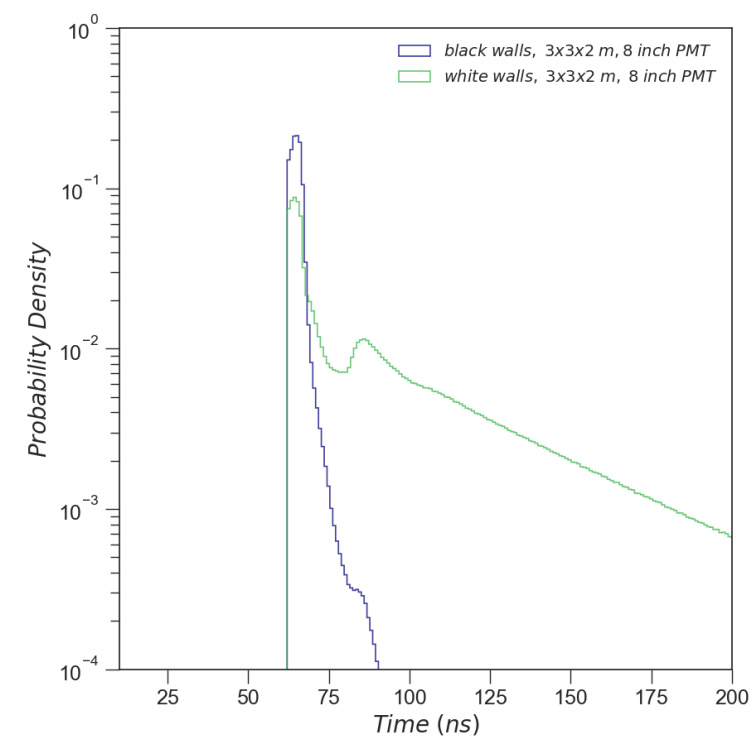

Figure 5: Timing distribution of a $3 \times 3 \times 2 \mathrm{~m}$ upper chamber of a WCD unit with $\gamma$-ray energy ranging from $1 \mathrm{MeV}$ to $1 \mathrm{GeV}$. The green and blue lines indicate a white-walled and black-walled chamber, respectively. sary given the long tail in the white-walled scenario (see Fig 5).

\subsection{Upper Chamber Dimension}

Vertical $\gamma$-rays with energies from 1 $\mathrm{MeV}$ to $1 \mathrm{GeV}$ were used to scan the surface of the upper chamber of WCD units of different dimensions and reflectivity, and compared to the HAWC configuration (see Fig. 6). The probability of detection between the white-walled chamber of $3 \times 3 \times 2$ $\mathrm{m}$ dimensions is almost double that of the HAWC configuration at lower energies $(\leq$ $100 \mathrm{MeV}$ ). However, at higher energies with a shallow chamber, there is a probability that the $\gamma$-rays do not cascade, and subsequently, no Cherenkov photons arrive at an 8 inch PMT. A deeper chamber of $3 \times 3 \times 3 \mathrm{~m}$ solves this problem at the cost of slightly reducing sensitivity below $\sim 25 \mathrm{MeV}$.

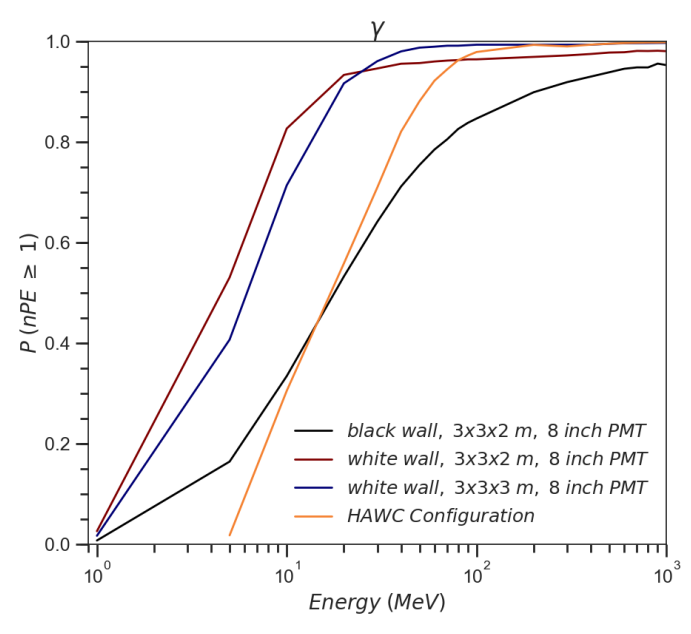

Figure 6: Probability of detection of one or more p.e in the upper chamber of a WCD unit with $\gamma$-ray energy ranging from $1 \mathrm{MeV}$ to $1 \mathrm{GeV}$ for various $\mathrm{WCD}$ designs. 

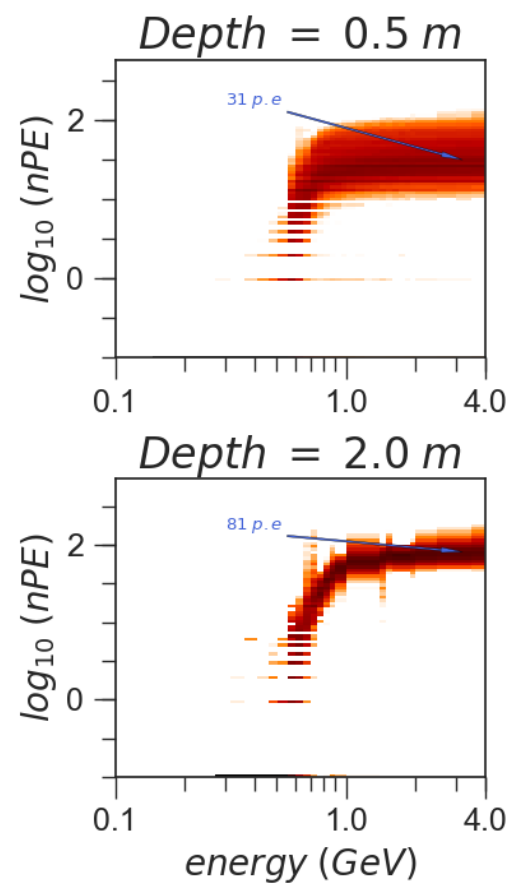
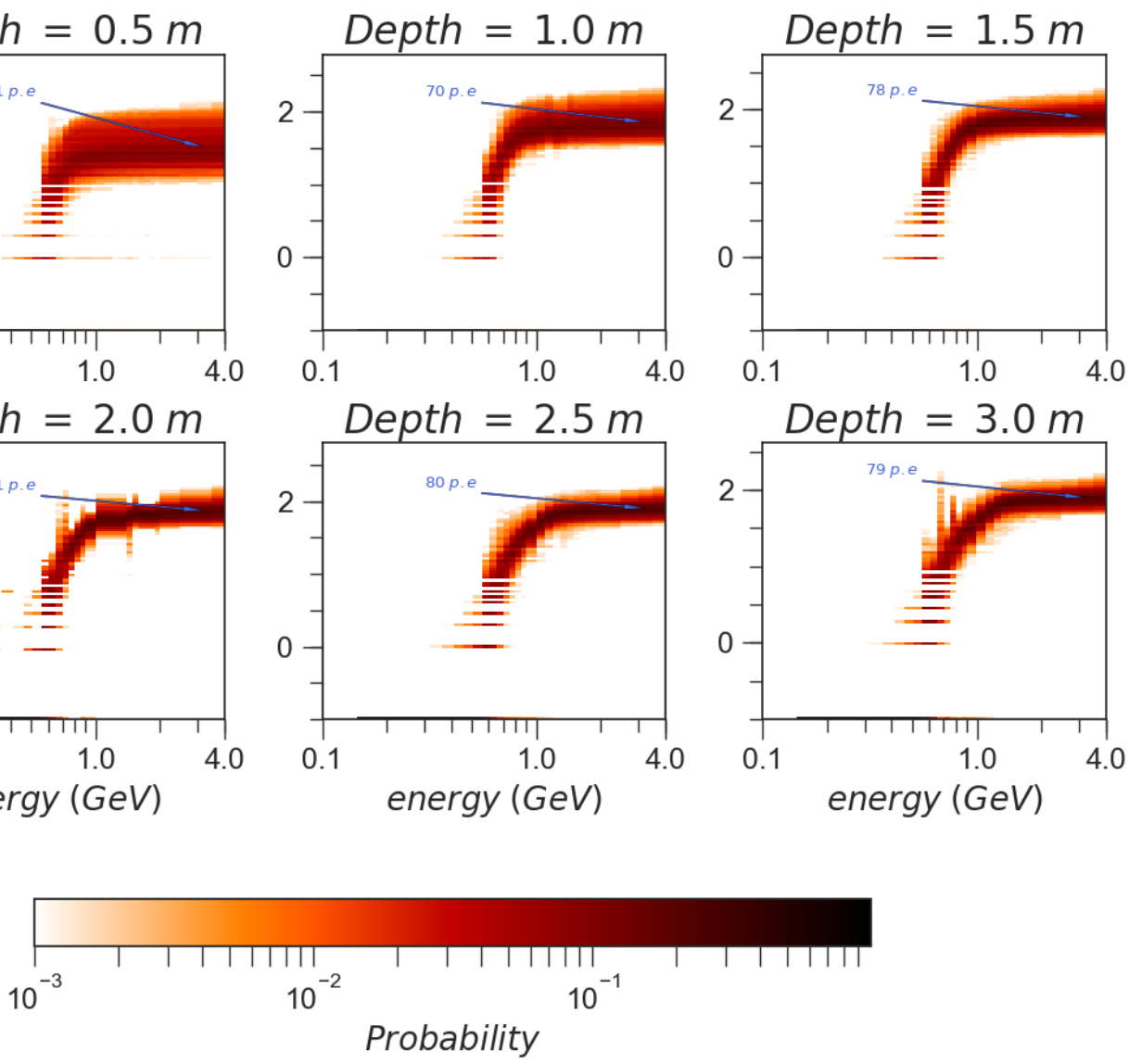

Figure 7: The number of p.e in a WCD unit with a white-walled $3 \times 3 \mathrm{~m}$ lower cell of various depths with $\mu$ energy ranging from $100 \mathrm{MeV}$ to $4 \mathrm{GeV}$.

Additionally, vertical $\gamma$-rays with energies from $10 \mathrm{MeV}$ to $2 \mathrm{GeV}$ in a WCD unit with upper chamber dimension of $3 \times 3 \times 2 \mathrm{~m}$ and lower chamber dimension of $3 \times 3 \times 1 \mathrm{~m}$ is compared with vertical $\mu$ - with energies from $100 \mathrm{MeV}$ to $4 \mathrm{GeV}$ in an identical WCD unit (see Fig 8). Muon identification is possible as the number of p.e detected in the two chambers remain constant above some energy threshold.

Furthermore, for very high $\gamma$-ray energies, the upper PMT can saturate as the particle density is very high. These $\gamma$-rays penetrate the lower chamber and can thus be used to get a reasonable estimate of the particle energy and thereby increase the dynamic range (see Fig 9). A 3 inch PMT in the lower chamber may be sufficient in this scenario. 

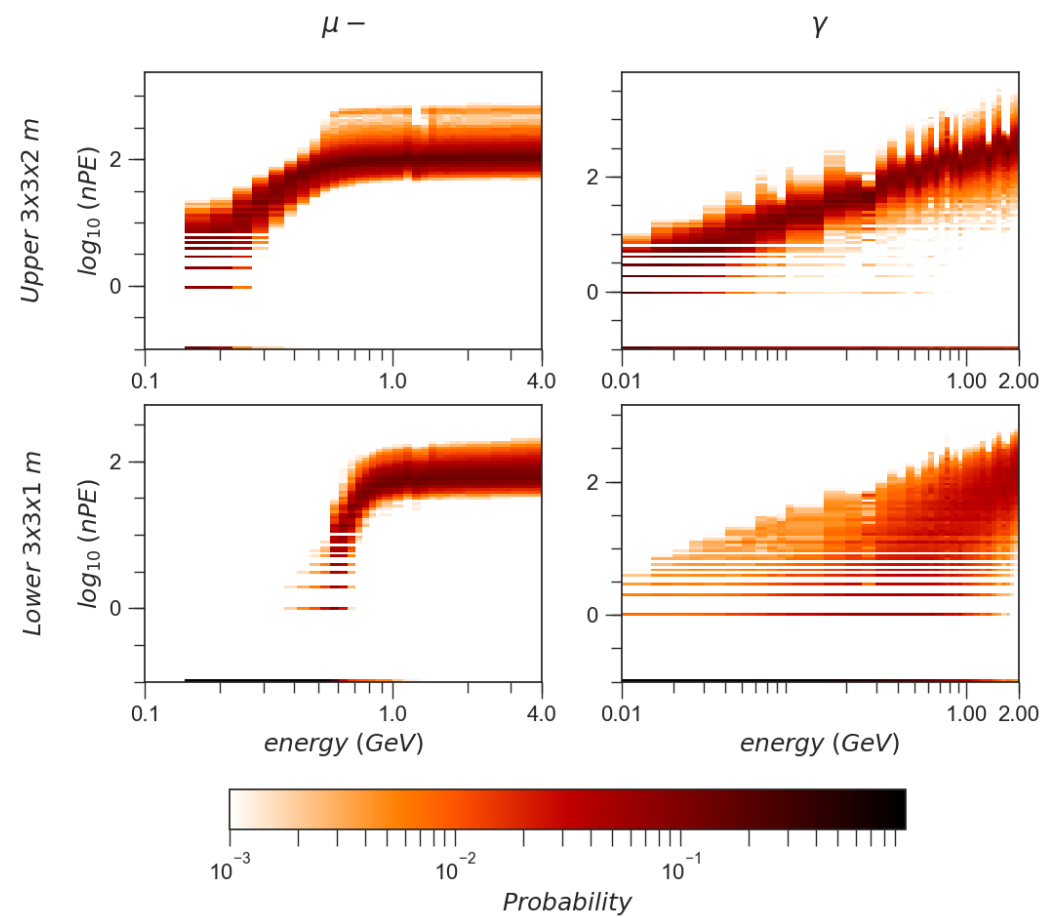

Figure 8: The number of p.e in a WCD unit with a white-walled $3 \times 3 \times 2 \mathrm{~m}$ upper and $3 \times 3 \times 1 \mathrm{~m}$ lower chamber with $\mu$ - energy ranging from $100 \mathrm{MeV}$ to $4 \mathrm{GeV}$ on the left and $\gamma$-ray energy ranging from $10 \mathrm{MeV}$ to 2 $\mathrm{GeV}$ on the right.

\subsection{Gamma-hadron separation}

Photons deposited aid in muon identification and subsequent gamma-hadron separation as the muon content is much higher and easily identifiable in hadronic cascades compared to those initiated by $\gamma$-rays as shown in Fig 10 for a WCD unit with an upper chamber of $3 \times 3 \times 3 \mathrm{~m}$ and lower chamber of $3 \times 3 \times 1.5 \mathrm{~m}$ dimensions. Here 10,000 vertical $5000 \mathrm{GeV}$ proton and $2000 \mathrm{GeV} \gamma$-ray initiated showers have been simulated.

\section{Summary \& Outlook}

We have begun the process of optimizing a WCD-based concept detector to improve sensitivity in the sub-TeV $\gamma$-ray energy range. Each detector unit comprises two

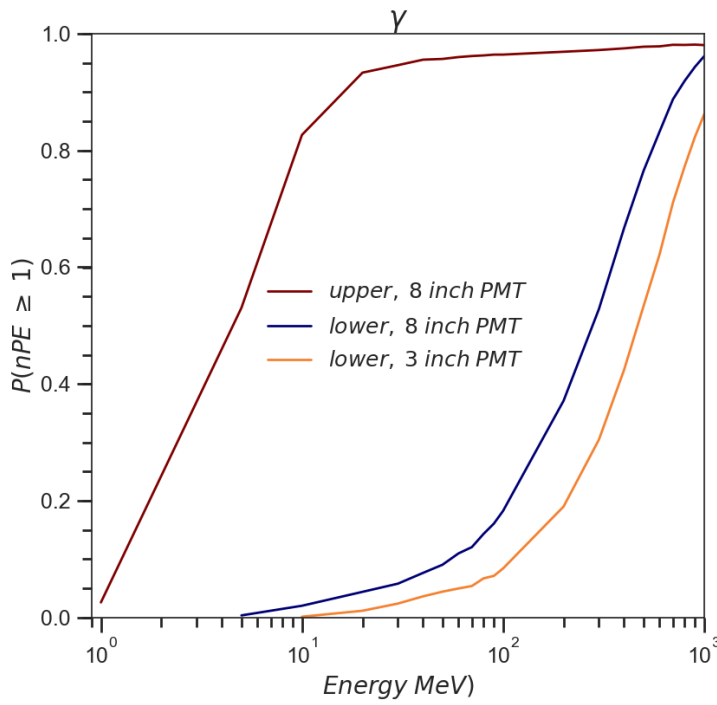

Figure 9: Probability of detection of one or more p.e in a WCD unit with a white $3 \times 3 \times 2 \mathrm{~m}$ upper and $3 \times 3 \times 1$ $\mathrm{m}$ lower chamber with $\gamma$-ray energy ranging from 1 $\mathrm{MeV}$ to $1 \mathrm{GeV}$. The dynamic range when the upper chamber is saturated recovers in the lower chamber for sufficiently high $\gamma$-ray energies. chambers with reflective wall linings and a PMT in each chamber. The upper PMT facing upwards is intended for timing and energy while the lower PMT facing downwards will enable muon tagging and increasing dynamic range. 

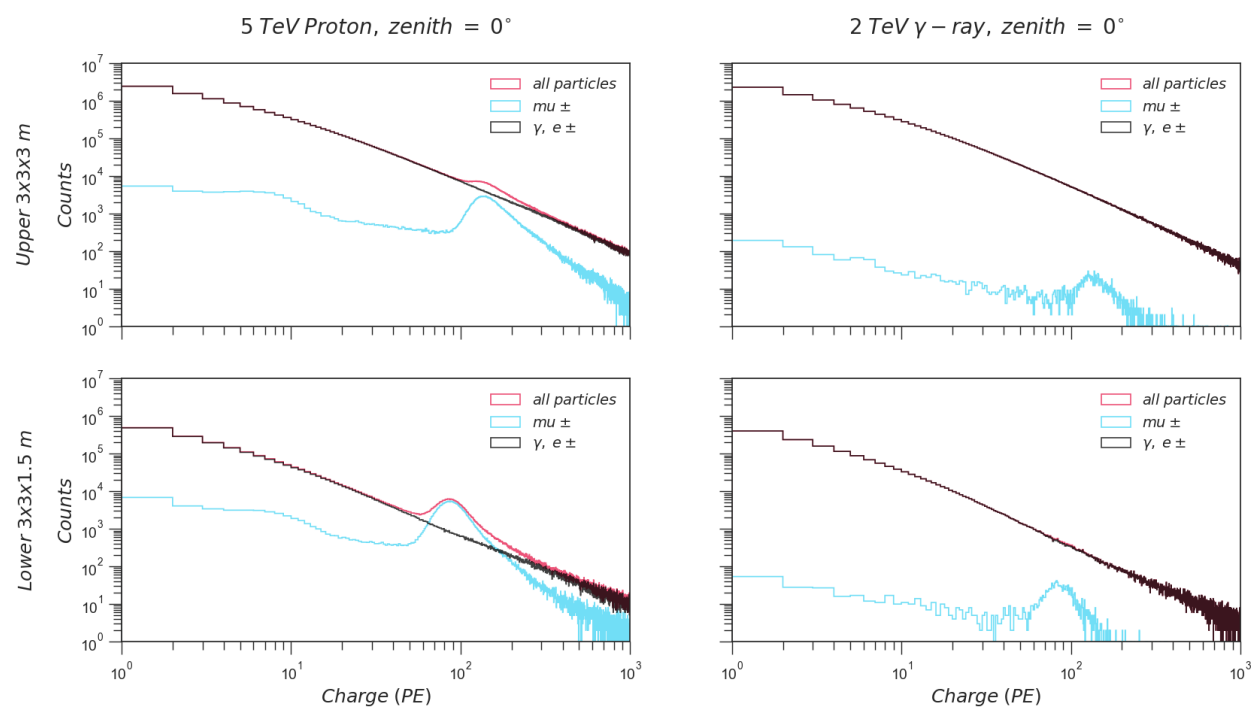

Figure 10: Charge distribution for all particles, electromagnetic particles and muons from vertical $5 \mathrm{TeV}$ protons (left) and $2 \mathrm{TeV} \gamma$-rays (right) arriving at the upper (top) and lower chamber (bottom) of WCD units of $3 \times 3 \times 3 \mathrm{~m}$ and $3 \times 3 \times 1.5 \mathrm{~m}$ dimensions respectively. A clear peak associated with well contained muons is visible.

We are investigating different options for chamber dimensions, reflectivity, and PMT sensitive area in the WCD units. A compact and highly reflective upper chamber should lower the energy threshold for the impinging particles and in conjunction with a lower chamber should improve gamma-hadron separation and air shower reconstruction.

\section{References}

[1] A. U. Abeysekara et al. (HAWC Collaboration), Astrophys. J. 843 (2017) 39.

[2] G. D. Sciascio et al. (LHAASO Collaboration), Nucl. Part. Phys. Proc. 279-281 (2016) 166-173.

[3] A. Albert et al. (SGSO Alliance), arXiv:1902.08429 (2019).

[4] Heck, D., et al., CORSIKA: a Monte Carlo code to simulate extensive air showers. (FZKA 6019)(Karlsruhe: Forschungszentrum Karlsruhe) (1998).

[5] S. Agostinelli et al., Nucl. Instrum. Methods Phys. Res. A 506 (2003) 205-303.

[6] H. Schoorlemmer et al., Eur. Phys. J. C 79 (2019) 427. https://doi.org/10.1140/epjc/s10052-019-6942-x

[7] S. Ostapchenko, Phys. Rev. D 83, 014018 (2011). https://doi.org/10.1103/PhysRevD.83.014018

[8] T.T. Bohlen et al., Nuclear Data Sheets 120, (2014) 211.

[9] A. Ferrari et al., CERN-2005-010, SLAC-R-773, INFN-TC-05-11 (2005).

[10] I. Allekotte et al. (LHAASO Collaboration), Nuclear Instruments and Methods in Physics Research A 586 (2008) 409-420.

[11] W.R. Nelson et al., (United States, 1986). https://www.osti.gov/servlets/purl/6800853 